\title{
Anterior leaflet splitting during transcatheter mitral valve replacement: Killing two birds with one stone?
}

\author{
Oliver J. Liakopoulos, MD, and Thorsten Wahlers, MD
}

\author{
From the Department of Cardiothoracic Surgery, Heart Center, University of Cologne, Cologne, Germany. \\ Disclosures: Authors have nothing to disclose with regard to commercial support. \\ Received for publication Nov 12, 2017; accepted for publication Nov 15, 2017; available ahead of print Jan 13, \\ 2018. \\ Address for reprints: Oliver J. Liakopoulos, MD, Department of Cardiothoracic Surgery, Heart Center of the Uni- \\ versity of Cologne, Kerpener Strasse. 62, 50924 Cologne, Germany (E-mail: oliver.liakopoulos@uk-koeln.de). \\ J Thorac Cardiovasc Surg 2018;155:e99-100 \\ $0022-5223 / \$ 36.00$ \\ Copyright (C) 2017 by The American Association for Thoracic Surgery \\ https://doi.org/10.1016/j.jtcvs.2017.11.055
}

Thanks to the dynamic evolution of surgical repair techniques and the introduction of less invasive and even robotic strategies, perioperative complication rates and long-term durability of mitral valve (MV) surgery have constantly improved in the past decades. ${ }^{1}$ Consistent with this evolution, the recent focused update of the 2014 American Heart Association and American College of Cardiology guidelines for the management of patients with valvular heart disease still recommends surgical repair or replacement as the preferred treatment for primary or secondary $\mathrm{MV}$ disease. $^{2}$

Encouraged by the excellent results of transcatheter aortic valve replacement and the early clinical experience with the MitraClip device (Abbott Vascular, Santa Clara, Calif), several transcatheter MV repair and MV replacement (TMVR) technologies that will most certainly redefine the future of MV therapy are currently being introduced. ${ }^{3}$ Importantly, the growing expertise of the heart valve team doing transcatheter MV interventions in patients judged not to be operative candidates has led to significant advancements during the past years. ${ }^{4}$ Nonetheless, several important and MV-specific challenges still need to be resolved, especially for TMVR, including the optimal access route, anchoring site, and positioning of the transcatheter valve to avoid paravalvular leaks or left ventricular outflow tract obstruction (LVOTO). ${ }^{5,6}$

Indeed, LVOTO after TMVR poses a significant clinical problem. Several predictors, including septal hypertrophy, a small left ventricular cavity, an aorta-mitral-annular angle (AMA) of less than $135^{\circ}$, and a length of the anterior MV leaflet (AML) greater than $30 \mathrm{~mm}$ have been previously identified. ${ }^{6}$ Thus, exact periprocedural imaging of the underlying anatomy of the MV and the left ventricular outflow tract seems mandatory for choosing the optimal TMVR strategy.

In this issue of the Journal, Lee and colleagues ${ }^{7}$ from St Louis describe a novel technique to prevent LVOTO during transapical TMVR that was used in a high-risk patient with case.

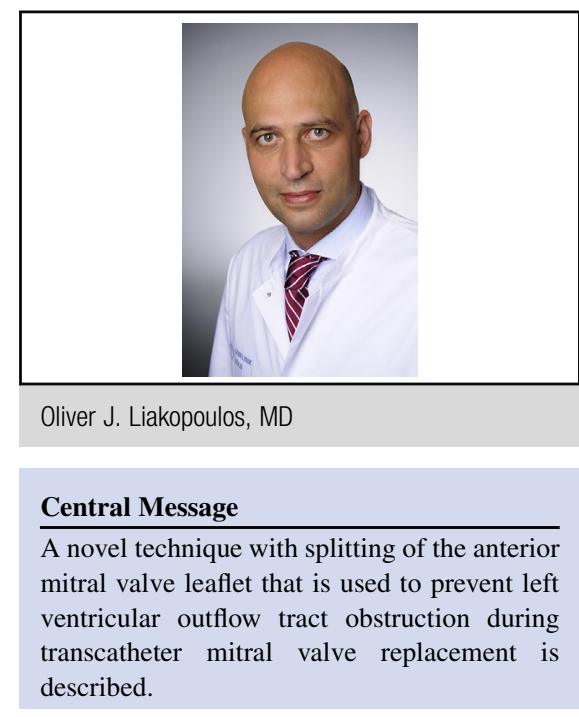

See Article page e95.

a history of previous MV repair and coronary artery bypass grafting. Preprocedural imaging revealed an AML length of $28 \mathrm{~mm}$, an aorta-mitral-annular angle of $110^{\circ}$, and the need for a $29-\mathrm{mm}$ valve size, thus rendering the patient susceptible to LVOTO after TMVR. Comparable to the chordalsparing MV surgical technique with AML detachment, the heart valve team from St Louis have introduced a novel, modified transcatheter AML splitting technique with subsequent TMVR. Echocardiographically and fluoroscopically guided puncture of the A2 segment of the AML and subsequent balloon-triggered AML detachment led to a severe mitral regurgitation, which was safely bridged by peripheral cardiopulmonary bypass before successful transapical valve deployment of a 29-mm Sapien valve (Edwards Lifesciences Corporation, Irvine, Calif). The heart valve team from St Louis are to be congratulated for their work, which seems to be justified by the satisfactory outcome of the presented

Two important things can be learned by this report. First, Lee and colleagues ${ }^{7}$ have added another valuable tool to the TMVR armamentarium by evolving an old surgery-based technique into a novel transcatheter approach. Although Lee and colleagues ${ }^{7}$ correctly highlight in their discussion that the AML splitting technique should be avoided in the presence of a calcified AML or in patients with previous MitraClip repair, this technique still has the potential to 
become an excellent option for patients with previous surgical MV repair who are not operative candidates and are at risk for LVOTO. Second, the well-balanced approach of Lee and colleagues, ${ }^{7}$ with meticulous preprocedural imaging and temporary cardiopulmonary bypass support during AML splitting and TMVR, underscores the exceptional ability of a well-structured heart valve team to enable a less invasive, safe treatment of complex MV pathologies in patients who would otherwise not be able to undergo intervention.

Whether the proposed transcatheter AML splitting technique will finally resolve the issue of LVOTO after TMVR needs to be evaluated in larger patient cohorts. Most importantly, however, the pursuit of more clearly defined periprocedural risk factors for LVOTO in TMVR needs to be continued so that we can identify patients who may benefit from this novel technique.

\section{References}

1. Woo YJ, Goldstone AB. The contemporary evolution of mitral valve surgery. $J$ Thorac Cardiovasc Surg. 2016;151:7-9.

2. Nishimura RA, Otto CM, Bonow RO, Carabello BA, Erwin JP III, Fleisher LA, et al. 2017 AHA/ACC focused update of the 2014 AHA/ACC guideline for the management of patients with valvular heart disease: a report of the American College of Cardiology/American Heart Association Task Force on Clinical Practice Guidelines. Circulation. 2017;135:e1159-95.

3. Badhwar V, Thourani VH, Ailawadi G, Mack M. Transcatheter mitral valve therapy: the event horizon. J Thorac Cardiovasc Surg. 2016;152:330-6.

4. Yoon SH, Whisenant BK, Bleiziffer S, Delgado V, Schofer N, Eschenbach L, et al. Transcatheter mitral valve replacement for degenerated bioprosthetic valves and failed annuloplasty rings. J Am Coll Cardiol. 2017;70:1121-31.

5. Testa L, Latib A, Montone RA, Bedogni F. Transcatheter mitral valve regurgitation treatment: state of the art and a glimpse to the future. J Thorac Cardiovasc Surg. 2016;152:319-27.

6. Bapat V, Pirone F, Kapetanakis S, Rajani R, Niederer S. Factors influencing left ventricular outflow tract obstruction following a mitral valve-in-valve or valvein-ring procedure, part 1. Catheter Cardiovasc Interv. 2015;86:747-60.

7. Lee R, Hui DS, Helmy TA, Lim MJ. Transapical mitral replacement with anterior leaflet splitting: a novel technique to avoid left ventricular outflow tract obstruction. J Thorac Cardiovasc Surg. 2018;155:e95-8. 